\title{
Knowledge, Attitude and Practice Regarding Blood Donation among Graduating Undergraduate Health Science Students at the University of Gondar, Northwest Ethiopia
}

\author{
Mulugeta Melku', Fikir Asrie ${ }^{1 *}$, Elias Shiferaw ${ }^{1}$, Berhanu Woldu ${ }^{1}$, Yalelet \\ Yihunew $^{2}$, Daniel Asmelash ${ }^{3}$, Bamlaku Enawgaw ${ }^{1}$
}

\section{OPEN ACCESS}

Citation: Mulugeta Melku, Fikir Asrie, Elias Shiferaw, et al. Knowledge, Attitude and Practice Regarding Blood Donation among Graduating Undergraduate Health Science Students at the University of Gondar, northwest Ethiopia. Ethiop J Health Sci.2018;28(5):571.

doi:http://dx.doi.org/10.4314/ejhs.v28i5.8

Received: September 1, 2017

Accepted: December 6, 2017

Published: September 1, 2018

Copyright: (C) 2018 Mulugeta Melku, et al. This is an open access article distributed under the terms of the Creative Commons Attribution License, which permits unrestricted use, distribution, and reproduction in any medium, provided the original author and source are credited.

Funding: Nil

Competing Interests: The authors declare that this manuscript was approved by all authors in its form and that no competing interest exists.

Affiliation and Correspondence:

${ }^{1}$ Department of Hematology and Immunohematology, School of Biomedical and Laboratory Sciences, College of Medicine and Health Sciences, University of Gondar, Gondar, Ethiopia

${ }^{2}$ School of Biomedical and Laboratory Sciences, College of Medicine and Health Sciences, University of Gondar, Gondar, Ethiopia ${ }^{3}$ Department of Clinical Chemistry, School of Biomedical and Laboratory Sciences, College of Medicine and Health Sciences, University of Gondar, Gondar, Ethiopia

*Email: fikirie2000@gmail.com

\section{ABSTRACT}

BACKGROUND: Blood transfusion is one of the most essential needs to manage patients suffering from various medical conditions. Nowadays, voluntary blood donors are the only source of blood in the blood banks. There is a great need to create awareness among the population at large and students about blood donation to maintain a regular blood supply. Health Science students can be used as best model to lead this initiative. Hence, the aim of this study was to assess the knowledge, attitude and practice regarding blood donation among graduating undergraduate Health Science students.

METHODS: $A$ descriptive cross-sectional study was conducted among graduating undergraduate Health Science students at University of Gondar using structured pre-tested self-administered questionnaire. Stratified sampling technique was employed to select study participants. A total of 225 students participated in the study. Data was entered into and analyzed using SPSS software version 20. Mean score was used to categorize the knowledge and attitude. Binary logistic regression model was fitted to identify factors associated with knowledge, attitude and practice regarding blood donation.

RESULT: Among 255 undergraduate Health Science graduate students, 123(48.2\%) and 202(79.2\%) had adequate knowledge about and positive attitude regarding blood donation, respectively. About 12.5\% of them had ever donated blood before. Age $\geq 25$ years was significantly associated with practice of blood donation (AOR=4.33; 95\%CI: 1.60, 11.76).

CONCLUSION: Although the majority of the students had positive attitude regarding blood donation, blood donation practice was low. Age was found to be significantly associated with blood donation practice. Targeted strategies should be designed to increase awareness of health science students about blood donation. Strategies which encourage the students to donate blood voluntarily should also be designed. KEYWORDS: Knowledge, attitude, practice, blood donation

INTRODUCTION 
Human blood is an important component of human life, and there are no alternatives to blood components yet (1). Blood transfusion is the donation of blood or blood products from the donor into the bloodstream of the recipient. It is a life-saving scheme in both routine and emergency situations to replace blood cells or blood products lost through bleeding with accident injuries, surgical conditions, malignancies, pregnancy complications and other medical conditions $(1,2)$.

Although the sufficient knowledge about blood donation is estimated to be $60 \%$ in developing countries; blood donation rate in low-income countries is far less than that in middle- and high-income countries $(3,4)$. Blood donation rate was less than satisfactory due to misconceptions, poor knowledge and unfavorable attitude toward donation (5). In addition, sex, age and educational status were found as predictors of voluntary blood donation (5-8). Members of the Ethiopian Jewish Community showed an extremely limited intention to donate blood (9). Every year, $25 \%-40 \%$ of Ethiopian pregnant mothers die due to shortage of enough blood supply from blood donors (10). Therefore, ensuring the availability of safe blood at all health facilities could reduce maternal deaths, which makes sure that the lives of every pregnant mother will not be threatened in case of emergencies for lack of blood (11). Although the fact that the country's annual demand of blood was 250,000 units, the amount of blood collected from donors by 2014 was 88,000 units (10). Similarly, the North Gondar blood bank needs 7,000 units of blood per year. However, only 4,500 units per year were collected in 2016 (North Gondar blood bank, unpublished data, 2016).

In Ethiopia, an integrated strategy for voluntary blood donation and recruiting a sufficient number of safe blood donors are major challenges (11). This could be attributed to low students' knowledge, discouraging attitude and poor blood donation practice regarding voluntary blood donation. As the result of this, blood banks and blood transfusion centers are obliged to organize more frequent blood drives to maintain a regular blood supply and to adopt an approach for enhancing new blood donor recruitment and retention of donors. One of the four components of World Health Organization basic strategy to encourage global safety and minimize risks associated with blood transfusion is that the blood should be collected only from voluntary donors $(2,4)$. Young population are crucial segment of the population, and they are the hope of present and future source of safe blood supply $(12,13)$. Part of the young population are Health Science university students who are healthy, active, dynamic, resourceful and receptive who may constitute a greater proportion to blood donation; and they have to be encouraged, inspired and motivated to donate blood voluntarily $(14,15)$. If appropriate strategies are designed and implemented to improve knowledge and attitude, health science students become not only the future blood donor but also the motivators plus the role models for the community. . Therefore, the main aim of this study was to assess knowledge, attitude and practice towards blood donation among graduating undergraduate Health Science students at the University of Gondar.

\section{MATERIALS AND METHODS}

Study area and period: A descriptive crosssection study was conducted on undergraduate Health Science graduating students from February to June 2014 at the University of Gondar. Gondar is found in North Gondar District, Amhara Regional State, northwest Ethiopia. Gondar town is located $738 \mathrm{Km}$ far from Addis Ababa to Northwest of Ethiopia. The University of Gondar has seven faculties: faculty of Business and Economics, Faculty of Social Science and Humanity, Faculty of Natural and Computational Sciences, Institute of Technology, Faculty of Agriculture, Faculty of Veterinary Medicine, and College of Medicine and Health Sciences. College of Medicine and Health Sciences encompasses teaching hospital, Institute of Public Health, School of Medicine, School of Pharmacy, School

DOI: http://dx.doi.org/10.4314/ejhs.v28i5.8 
of Biomedical and Laboratory Science, School of Nursing and Department of Midwifery. At the time of the study, the college had nine health science departments with graduating students.

Study population: The source populations were all graduating undergraduate Health Science students at University of Gondar who were voluntary to participate and be available during data collection period. Those students who were not voluntary to participate and those who critically ill were excluded from the study.

Sample size determination: Single population proportion formula, $\left[n=(Z \alpha / 2)^{2} p(1-p) / d^{2}\right]$, was used to calculate the sample size. Due to lack of similar published information showing the knowledge, attitude and practice of blood donation in this particular study area, $50 \%$ was used to get the maximum sample size by considering $95 \%$ confidence interval, marginal error $(d)$ of $5 \%$ and $10 \%$ non-respondent rate. Since the source population was less than ten thousand $(\mathrm{N}$ $<10,000$ ), sample size correction formula was applied, and then, the final sample size was determined to be 255 .

Sampling technique and procedure: Proportional stratified sampling method was employed for this study. To maintain relative homogeneity concerning our research interests, the students were grouped in to two strata. The strata are made by considering their education proximity to transfusion practice. The first stratum consisted of Health Officer, Anesthesia, Nursing, Midwifery and Medical Laboratory Science students. The second stratum encompassed Pharmacy, Optometry, Physiotherapy, Psychiatry and Environmental and Occupational Health and Safety students. Based on the number of students under each stratum, the samples to be studied were determined by proportional allocation method. Likewise, the number of students from each department under the stratum was determined by proportional allocation. Finally, using the sampling frame obtained from the Registrar Office, the study sample was selected by simple random sampling technique of lottery method (Figure 1).

Data collection technique: The data was collected using pre-tested, structured self-administered questionnaire. It includes questions on sociodemographic characteristics of students and questions which sought knowledge, attitude and practice regarding blood donation.

Assessment of knowledge, attitude and practice: Knowledge about blood donation was assessed using 20 questions. Each response was scored as " 1 " for correct response and " 0 " for incorrect response. The study participants who scored $70 \%$ and above for the knowledge assessment questions were considered as having adequate knowledge. Similarly, attitude towards blood donation was assessed using 11 questions. The responses were scored as " 1 " for positive attitude" and " 0 " for negative attitude for each attitude question. The study participants who scored $70 \%$ and above for the attitude assessment questions were considered as having positive attitude. Moreover, practice was assessed by asking about history of previous donation and the frequency of donation.

Data processing and analysis: The data was cleaned, edited and checked for completeness. Data were entered into Epi Info Version 3.1 and exported into SPSS for data processing and analysis. Then, the data were analyzed using appropriate descriptive and inferential statistical tests. Binary logistic regression model was used to identify factors associated with knowledge, attitude and practice concerning blood donation. Crude odds ratio (COR) and adjusted odds ratio (AOR) with their 95\% confidence interval (CI) were used to measure the strength of association between dependent and independent variables. $P$ value $<0.05$ was considered for statistical significance for all statistical tests. 


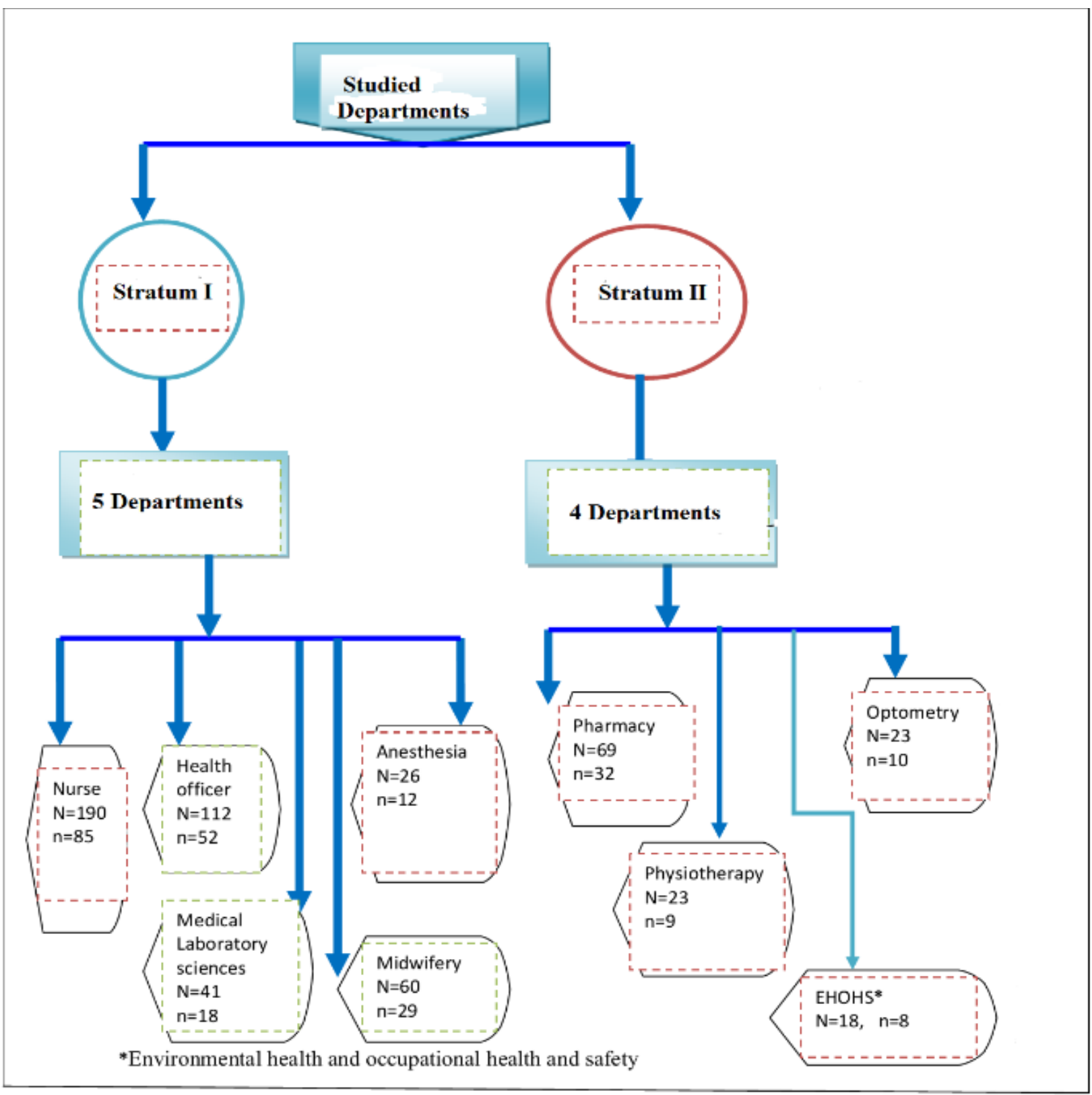

Figure 1:- Graphical representation of the sampling procedure

Ethical consideration: Ethical approval was obtained from the University of Gondar, School of Biomedical and Laboratory Sciences Ethical Clearance Committee. Each study participant was asked to participate voluntarily, after they had been informed about the objective of the study. They had also been informed that the participation was voluntary in which the participants had full right to withdraw at any time. When they agreed to participate, informed consent was obtained. Confidentiality of the participants' information was kept throughout the research processes.

DOI: http://dx.doi.org/10.4314/ejhs.v28i5.8 


\section{RESULTS}

Socio-demographic characteristics: A total of 255 graduating undergraduate Health Science students responded to the questionnaire, with a response rate of $100 \%$. This $100 \%$ response rate was achieved since the data collection facilitators were experienced and the issue of blood donation is not sensitive. As a result, the participants did not fail to comply with the intended study. The majority of the study participants, $227(89 \%)$, were within the age range of 20-24 years, and $211(82.7 \%)$ of them were males. Moreover, 249 $(97.6 \%), 229(89.8 \%)$, and $175(68.6 \%)$ of the respondents were single by marital status, Orthodox Christians by religion and Amhara by ethnicity, respectively (Table 1).

Table 1. Characteristics of graduating undergraduate Health Science students at University of Gondar, 2014.

\begin{tabular}{|c|c|c|c|}
\hline Variables & Category & $\#$ & $\%$ \\
\hline \multirow[t]{2}{*}{ Sex } & Male & 211 & 82.7 \\
\hline & Female & 44 & 17.3 \\
\hline \multirow[t]{2}{*}{ Residence } & Rural & 164 & 64.3 \\
\hline & Urban & 91 & 35.7 \\
\hline \multirow[t]{2}{*}{ Age } & 20-24 year & 227 & 89 \\
\hline & $\geq 25$ year & 28 & 11 \\
\hline \multirow{4}{*}{ Religion } & Orthodox & 229 & 89.8 \\
\hline & Muslim & 19 & 7.5 \\
\hline & Protestant & 7 & 2.7 \\
\hline & Don't have & 136 & 53.3 \\
\hline \multirow[t]{2}{*}{ Marital status } & Single & 249 & 97.6 \\
\hline & Married & 6 & 2.4 \\
\hline \multirow[t]{9}{*}{ Department } & Health officer & 52 & 20.4 \\
\hline & Nursing & 85 & 33.3 \\
\hline & Midwifery & 29 & 11.4 \\
\hline & Anesthesia & 12 & 4.7 \\
\hline & Pharmacy & 32 & 12.5 \\
\hline & Laboratory technology & 18 & 7.1 \\
\hline & Physiotherapy & 9 & 3.5 \\
\hline & Environmental health & 8 & 3.1 \\
\hline & Optometry & 10 & 4.0 \\
\hline \multirow[t]{4}{*}{ Ethnicity } & Amhara & 175 & 68.6 \\
\hline & Tigray & 19 & 7.5 \\
\hline & Oromo & 12 & 4.7 \\
\hline & Other & 18 & 7.1 \\
\hline
\end{tabular}

'Other' includes Addis Ababa, SNNP, Somali and Afar

Knowledge of study participants about blood donation: Out of the total study participants, $123(48.2 \%)$ had adequate knowledge whereas $149(51.6 \%)$ had inadequate knowledge about blood donation. The majority, 234(91.8\%) and
$188(73.7 \%)$ of the study participants did not know the maximum age and the minimum weight to be eligible for blood donation, respectively (Table 2).

Table 2: Participants' response to knowledge assessment questions about blood donation. 


\begin{tabular}{|c|c|c|c|c|}
\hline \multirow[t]{2}{*}{ Knowledge Questions } & \multirow[t]{2}{*}{ Responses } & \multirow{2}{*}{$\begin{array}{l}\text { Frequency } \\
\text { n }(\%)\end{array}$} & \multicolumn{2}{|c|}{ Knowledge toward Blood donation } \\
\hline & & & $\begin{array}{l}\text { Correct response } \\
\mathrm{n}(\%)\end{array}$ & $\begin{array}{l}\text { Incorrect response } \\
\mathrm{n}(\%)\end{array}$ \\
\hline \multirow{3}{*}{$\begin{array}{l}\text { Is blood donation harmful to } \\
\text { donor? }\end{array}$} & Yes & $37(14.5)$ & & \\
\hline & No & $209(82)$ & $209(82)$ & $46(18)$ \\
\hline & No idea & $4(1.6)$ & & \\
\hline \multirow{5}{*}{$\begin{array}{l}\text { Where is the place of blood } \\
\text { donation? }\end{array}$} & Hospital & $143(56.1)$ & & \\
\hline & Health center & $15(5.9)$ & $180(70.6)$ & $75(29.5$ \\
\hline & Donation center & $37(14.5)$ & & \\
\hline & Red cross center & $56(22)$ & & \\
\hline & Other & $4(1.6)$ & & \\
\hline \multirow[t]{2}{*}{ Goal of blood donation } & Saving relatives' life & $58(22.7)$ & & \\
\hline & $\begin{array}{l}\text { Saving someone's life } \\
\text { getting insurance }\end{array}$ & $\begin{array}{l}193(75.7) \\
4(1.6)\end{array}$ & 193(75.5) & $62(24.3)$ \\
\hline \multirow[t]{2}{*}{ Minimum age to donate blood } & $>18$ or $<18$ years & $116(45.5)$ & & \\
\hline & 18 year & $139(54.5)$ & $139(54.5)$ & $116(45.5)$ \\
\hline \multirow[t]{4}{*}{ Maximum age to donate blood } & $<65$ year & $189(74.1)$ & & \\
\hline & 65 year & $21(8.2)$ & $21(8.2)$ & $234(91.8)$ \\
\hline & $>65$ year & $13(5.1)$ & & \\
\hline & Don't know & $32(12.6)$ & & \\
\hline \multirow{4}{*}{$\begin{array}{l}\text { What is the minimum weight for } \\
\text { blood donation }\end{array}$} & $<45 \mathrm{Kg}$ & $23(9)$ & & \\
\hline & $45 \mathrm{Kg}$ & $67(26.3)$ & $67(26.3)$ & $188(73.7)$ \\
\hline & $>45 \mathrm{Kg}$ & $148(58)$ & & \\
\hline & Don't know & $17(6.7)$ & & \\
\hline \multirow{4}{*}{$\begin{array}{l}\text { What is the maximum volume of } \\
\text { blood at once donation }\end{array}$} & $250 \mathrm{ml}$ & $39(15.3)$ & & \\
\hline & $350 \mathrm{ml}$ & $55(21.6)$ & $114(44.7)$ & $141(55.3)$ \\
\hline & $450 \mathrm{ml}$ & $114(44.7)$ & & \\
\hline & Don’t know & $47(18)$ & & \\
\hline \multirow{4}{*}{$\begin{array}{l}\text { At what minimum interval can a } \\
\text { person donate blood }\end{array}$} & Every 3 month & $173(67.8)$ & & \\
\hline & Every 6 month & $43(16.9)$ & $173(67.8)$ & $82(32.2)$ \\
\hline & Once in a year & $6(2.4)$ & & \\
\hline & Don’t know & $33(12)$ & & \\
\hline \multirow[t]{2}{*}{ Do you know about blood group } & Yes & $233(91.4)$ & 233(91.4) & $22(8.2)$ \\
\hline & No & $22(8.6)$ & & \\
\hline \multirow{5}{*}{$\begin{array}{l}\text { What is the most common blood } \\
\text { group type }\end{array}$} & A & $51(20)$ & & \\
\hline & B & $15(5.9)$ & $96(37.6)$ & $159(62.4)$ \\
\hline & $\mathrm{AB}$ & $92(36.1)$ & & \\
\hline & $\mathrm{O}$ & $96(37.6)$ & & \\
\hline & Don't know & $1(0.4)$ & & \\
\hline \multirow{3}{*}{$\begin{array}{l}\text { Can pregnant women donate } \\
\text { blood? }\end{array}$} & Yes & $20(7.8)$ & & \\
\hline & No & $234(91.8)$ & $234(91.8$ & $21(8.2)$ \\
\hline & Don't know & $1(0.4)$ & & \\
\hline \multirow{3}{*}{$\begin{array}{l}\text { Can women Female during } \\
\text { menstruation donate blood? }\end{array}$} & Yes & $52(20.4)$ & & \\
\hline & No & $202(79.2)$ & $202(79.2)$ & $53(20.8)$ \\
\hline & Don't know & $1(0.4)$ & & \\
\hline
\end{tabular}

DOI: http://dx.doi.org/10.4314/ejhs.v28i5.8 
Table 2. Continued...

\begin{tabular}{|c|c|c|c|c|}
\hline \multirow{3}{*}{$\begin{array}{l}\text { Can cigarette smokers donate } \\
\text { blood? }\end{array}$} & Yes & $97(38)$ & & \\
\hline & No & $92(36.1)$ & $92(36.1)$ & $163(63.9)$ \\
\hline & Don't know & $66(25.9)$ & & \\
\hline \multirow{3}{*}{$\begin{array}{l}\text { Person can be infected by } \\
\text { receiving blood transfusion }\end{array}$} & Yes & $209(81)$ & & \\
\hline & No & $42(16.5)$ & $209(82)$ & $46(18)$ \\
\hline & Don't know & $4(1.6)$ & & \\
\hline \multirow{3}{*}{$\begin{array}{l}\text { Can a person donate when } \\
\text { blood pressure is low }\end{array}$} & Yes & $38(14.9)$ & & \\
\hline & No & $196(76.9)$ & $196(76.9)$ & $59(23.1)$ \\
\hline & Don’t know & $21(8.2)$ & & \\
\hline \multirow{3}{*}{$\begin{array}{l}\text { Can a person with high blood } \\
\text { pressure donate blood }\end{array}$} & Yes & $94(36.9)$ & & \\
\hline & No & $129(50.6)$ & $129(50.6)$ & $126(49.40)$ \\
\hline & Don’t know & $32(12.5)$ & & \\
\hline \multirow{2}{*}{$\begin{array}{l}\text { Can HIV infected person } \\
\text { donate blood? }\end{array}$} & Yes & $23(9)$ & & \\
\hline & $\begin{array}{l}\text { No } \\
\text { don't know }\end{array}$ & $\begin{array}{l}226(88.6) \\
6(2.4)\end{array}$ & $226(88.6)$ & $29(11.4)$ \\
\hline \multirow{5}{*}{$\begin{array}{l}\text { Disease that can be transmitted } \\
\text { by transfusion } \\
\text { Best source of donor blood }\end{array}$} & $\mathrm{HBV}, \mathrm{HCV}$, malaria & $193(75.7)$ & & \\
\hline & TB, Don’t know & $62(24.3)$ & $193(75.7)$ & $62(24.3)$ \\
\hline & Voluntary & $234(91.8)$ & & \\
\hline & $\begin{array}{l}\text { Replacement, } \\
\text { remunerated }\end{array}$ & $14(5.47)$ & $234(91.8)$ & $21(8.2)$ \\
\hline & Don't know & $7(2.73)$ & & \\
\hline \multirow{3}{*}{$\begin{array}{l}\text { Do all surgical procedure } \\
\text { requires blood transfusion }\end{array}$} & Yes & $26(10.1)$ & & \\
\hline & No & $222(87.1)$ & $222(87.1)$ & $33(12.9)$ \\
\hline & No idea & $7(2.7)$ & & \\
\hline
\end{tabular}

Attitude of study participants regarding blood donation: The majority, 202(79.2\%), of the respondents had positive attitude whereas the remaining 53(20.8\%) had negative attitude about blood donation. About 220(86.3\%) of respondents believed that blood donation is a moral duty, and $218(85.5 \%)$ of them were voluntary to donate blood for the future (Table 3 ).

DOI: http://dx.doi.org/10.4314/ejhs.v28i5.8 
Table 3: Participants' responses to selected attitude assessment questions about blood donation.

\begin{tabular}{|c|c|c|c|c|}
\hline \multirow[t]{2}{*}{ Attitude Questions } & \multirow[t]{2}{*}{ Responses } & \multirow{2}{*}{$\begin{array}{l}\text { Frequency } \\
(\%)\end{array}$} & \multicolumn{2}{|c|}{ Attitude regarding Blood donation } \\
\hline & & & $\begin{array}{l}\text { positive attitude } \\
\text { n }(\%)\end{array}$ & $\begin{array}{l}\text { Negative attitude } \\
\mathrm{n}(\%)\end{array}$ \\
\hline \multirow{3}{*}{$\begin{array}{l}\text { blood donation is moral } \\
\text { duty }\end{array}$} & Yes & $220(86.3)$ & \multirow{3}{*}{$220(86.3)$} & \multirow{3}{*}{$35(13.7)$} \\
\hline & No & $26(10.2)$ & & \\
\hline & No idea & $9(3.5)$ & & \\
\hline \multirow{3}{*}{$\begin{array}{l}\text { Willingness to donate blood } \\
\text { For the future }\end{array}$} & Yes & $218(85.5)$ & \multirow{3}{*}{$218(85.5)$} & \multirow{3}{*}{$37(14.5)$} \\
\hline & No & $17(6.7)$ & & \\
\hline & No idea & $20(2.8)$ & & \\
\hline \multirow{3}{*}{$\begin{array}{l}\text { Willingness to donate blood } \\
\text { to unknown person if asked }\end{array}$} & Yes & $187(73.3)$ & \multirow{3}{*}{$187(73.3)$} & \multirow{3}{*}{$68(26.7)$} \\
\hline & No & $47(18.4)$ & & \\
\hline & No idea & $21(8.2)$ & & \\
\hline \multirow{3}{*}{$\begin{array}{l}\text { Do you encourage others to } \\
\text { donate blood }\end{array}$} & Yes & $227(89)$ & \multirow{3}{*}{$227(89)$} & \multirow{3}{*}{$20(11)$} \\
\hline & No and & $20(7.9)$ & & \\
\hline & No Idea & $8(3.1)$ & & \\
\hline \multirow{3}{*}{$\begin{array}{l}\text { Willingness to become } \\
\text { regular donor }\end{array}$} & Yes & $85(33.3)$ & \multirow{3}{*}{$85(33.3)$} & \multirow{3}{*}{$170(66.7)$} \\
\hline & No & $138(54.2)$ & & \\
\hline & No idea & $32(12.5)$ & & \\
\hline \multirow{3}{*}{$\begin{array}{l}\text { Willingness to tell family if } \\
\text { donated blood }\end{array}$} & Yes & $194(76.1)$ & \multirow{3}{*}{ 194(76.1) } & \multirow{3}{*}{$61(23.9)$} \\
\hline & No & $48(18.8)$ & & \\
\hline & No idea & $13(5.1)$ & & \\
\hline
\end{tabular}

Practice of blood donation: About 32 (12.5\%) of the respondents reported that they had ever donated blood at least once. A larger proportion, $28(87.5 \%)$, of the participants who ever donated blood reported that they achieved satisfaction after donation. The majority, 223(87.5\%), of the study

Table 4: Practice of blood donation, and reasons for donating and not-donating blood.

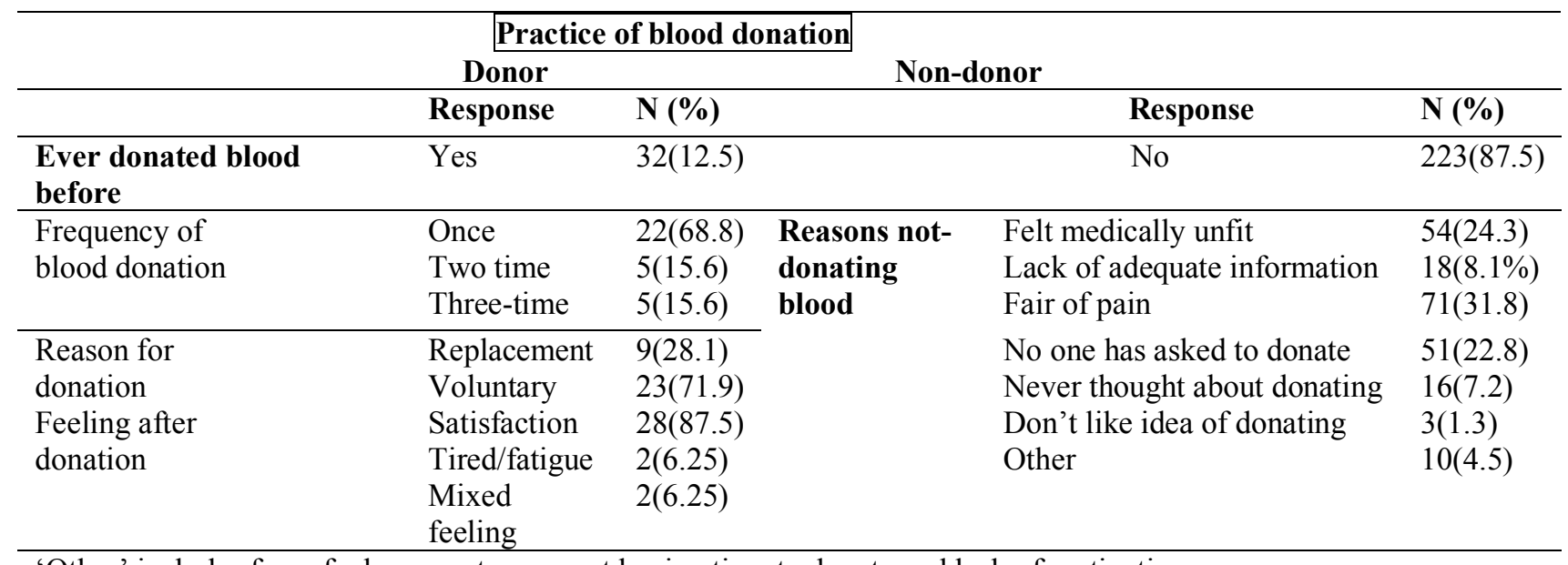

'Other' includes fear of adverse outcome, not having time to donate and lack of motivation

participants had never donated blood before. Their reasons were fear of pain, 71(31.8\%), feeling of medical unfitness, 54(24.3\%) and not having been asked to donate blood, 51(22.8\%) (Table 4). 
Factors associated with knowledge, attitude and practice: Among the socio-demographic variables, age of the study participants was significantly associated with practice of blood donation. Accordingly, students aged $\geq 25$ years were about four times $(\mathrm{AOR}=4.33 ; 95 \% \mathrm{CI}: 1.60$, 11.76) more likely to donate blood compared to students aged 20-24 years (Table 5). However, none of these factors were associated with knowledge and attitude regarding blood donation.

Table 5: Association of socio-demographic characteristics of study participants with Knowledge, Attitude and Practice toward blood donation.

\begin{tabular}{|c|c|c|c|c|c|}
\hline \multicolumn{6}{|c|}{ Knowledge about blood donation versus sociodemographic characteristic of study participants } \\
\hline Variable & Response & $\begin{array}{l}\text { knowledgeable } \\
\text { N (\%) }\end{array}$ & $\begin{array}{l}\text { Not knowledgeable } \\
\text { N (\%) }\end{array}$ & COR (95\%CI) & AOR (95\%CI) \\
\hline \multirow[t]{2}{*}{ Sex } & Male & $100(47.4)$ & $111(52.6)$ & $0.82(0.48,1.58)$ & \\
\hline & Female & $23(52.3)$ & $21(47.7)$ & 1.00 & \\
\hline \multirow{2}{*}{ Age in years } & $20-24$ & $107(47.1)$ & $120(52.9)$ & $0.67(0.3,1.48)$ & \\
\hline & $>=25$ & $16(57.1))$ & $12(42.9)$ & 1.00 & \\
\hline \multirow[t]{2}{*}{ Marital status } & Single & $121(48.6)$ & $128(51.4)$ & $1.9(0.35,10.52)$ & \\
\hline & Married & $2(33.3)$ & $4(66.7)$ & 1.00 & \\
\hline \multirow[t]{2}{*}{ Place of birth } & Urban & $47(51.6)$ & $44(48.4)$ & 1.00 & \\
\hline & Rural & $76(46.3)$ & $88(53.7)$ & $0.81(0.48,1.35)$ & \\
\hline \multirow{2}{*}{$\begin{array}{l}\text { Departmental } \\
\text { group }\end{array}$} & Group-I & $101(51.5)$ & $95(48.5)$ & 1.00 & \\
\hline & Group-II & $22(37.3)$ & 37 (62.7) & $0.56(0.31,1.02)$ & \\
\hline \multicolumn{6}{|c|}{ Attitude towards blood donation versus socio-demographic characteristic of study participants } \\
\hline Variable & Response & $\begin{array}{l}\text { Positive attitude } \\
\text { N (\%) }\end{array}$ & $\begin{array}{l}\text { Poor attitude } \\
\text { N }(\%)\end{array}$ & COR (95\%CI) & $\operatorname{AOR}(95 \% \mathrm{CI})$ \\
\hline \multirow[t]{2}{*}{ Sex } & Male & $167(79.1)$ & $44(20.9)$ & $0.96(0.44,2.20)$ & \\
\hline & Female & $35(79.5)$ & $9(20.5)$ & 1.00 & \\
\hline \multirow[t]{2}{*}{ Age in years } & $20-24$ & $178(78.4)$ & $24(85.7)$ & $0.61(0.20,1.83)$ & \\
\hline & $>=25$ & $49(21.6))$ & $4(14.3)$ & 1.00 & \\
\hline \multirow[t]{2}{*}{ Marital status } & Single & $179(79.1)$ & $52(20.9)$ & $0.76(0.10,6.62)$ & \\
\hline & Married & $5(83.3)$ & $1(16.7)$ & 1.00 & \\
\hline \multirow[t]{2}{*}{ Place of birth } & Urban & $74(81.3)$ & $17(18.7)$ & 1.00 & \\
\hline & Rural & $128(78)$ & $36(22)$ & $0.82(0.43,1.60)$ & \\
\hline \multirow{2}{*}{$\begin{array}{l}\text { Departmental } \\
\text { group }\end{array}$} & Group-I & $157(80.1)$ & 39 (19.9) & 1.00 & \\
\hline & Group-II & $45(76.3)$ & $14(23.7)$ & $0.80(0.40,1.60)$ & \\
\hline \multicolumn{6}{|c|}{ Practice towards blood donation versus socio-demographic characteristic study participants } \\
\hline \multirow{3}{*}{$\begin{array}{l}\text { Vari } \\
\text { Sex }\end{array}$} & Response & Donor N (\%) & Non-donor N (\%) & COR $(95 \% \mathrm{CI})$ & AOR(95\%CI) \\
\hline & Male & $23(10.9)$ & $188(89.1)$ & $0.48(0.20,1.12)$ & \\
\hline & Female & $9(20.5)$ & $35(79.5)$ & 1.00 & \\
\hline \multirow[t]{2}{*}{ Age in years } & $20-24$ & $24(10.6)$ & $203(89.4)$ & 1.00 & \\
\hline & $>=25$ & $8(28.6))$ & $20(71.4)$ & $3.38(1.35,8.55)$ & $4.33(1.60,11.76)$ \\
\hline \multirow[t]{2}{*}{ Marital status } & Single & $31(12.4)$ & $218(87.6)$ & 1.00 & \\
\hline & Married & $1(16.7)$ & $5(83.3)$ & $1.4(0.16,12.5)$ & \\
\hline \multirow[t]{2}{*}{ Place of birth } & Urban & $14(15.4)$ & $77(84.6)$ & $1.47(0.703 .13)$ & \\
\hline & Rural & $18(11))$ & $146(89)$ & 1.00 & \\
\hline \multirow{2}{*}{$\begin{array}{l}\text { Departmental } \\
\text { group }\end{array}$} & Group-I & $24(12.2)$ & $172(87.8)$ & $1.12(0.50,2.65)$ & \\
\hline & Group-II & $8(13.6)$ & $51(86.4)$ & 1.00 & \\
\hline
\end{tabular}

Note: Departments group-I comprises Health officer, Anesthesia, Nurse, Midwifery and Medical Laboratory Sciences; Group-II comprises physiotherapy, Pharmacy, Optometry and EHOHS

DOI: http://dx.doi.org/10.4314/ejhs.v28i5.8 
Table 5: Practice of blood donation versus socio-demographic characteristic study participants

\begin{tabular}{|c|c|c|c|c|c|}
\hline \multirow[t]{2}{*}{ Variable } & \multirow[t]{2}{*}{ Response } & \multicolumn{2}{|c|}{ Practice of blood donation } & \multirow[t]{2}{*}{$\mathrm{COR}(95 \% \mathrm{CI})$} & \multirow[t]{2}{*}{$\operatorname{AOR}(95 \% \mathrm{CI})$} \\
\hline & & $\begin{array}{l}\text { Donor } \\
\mathrm{n}(\%)\end{array}$ & $\begin{array}{l}\text { Non-donor } \\
\mathrm{n}(\%)\end{array}$ & & \\
\hline Sex & Male & $23(10.9)$ & $188(89.1)$ & $0.48(0.20,1.12)$ & \\
\hline Age & $\begin{array}{l}\text { Female } \\
20-24 \text { years } \\
>=25 \text { year }\end{array}$ & $\begin{array}{l}9(20.5) \\
24(10.6) \\
8(28.6))\end{array}$ & $\begin{array}{l}35(79.5) \\
203(89.4) \\
20(71.4)\end{array}$ & $\begin{array}{l}1.00 \\
1.00 \\
3.38(1.35,8.55)\end{array}$ & $4.33(1.60,11.76)^{* * *}$ \\
\hline Marital status & $\begin{array}{l}\text { Single } \\
\text { Married }\end{array}$ & $\begin{array}{l}31(12.4) \\
1(16.7)\end{array}$ & $\begin{array}{l}218(87.6) \\
5(83.3)\end{array}$ & $\begin{array}{l}1.00 \\
1.4(0.16,12.5)\end{array}$ & \\
\hline Place of birth & $\begin{array}{l}\text { Urban } \\
\text { Rural }\end{array}$ & $\begin{array}{l}14(15.4) \\
18(11))\end{array}$ & $\begin{array}{l}77(84.6) \\
146(89)\end{array}$ & $\begin{array}{l}1.47(0.703 .13) \\
1.00\end{array}$ & \\
\hline $\begin{array}{l}\text { Departmental } \\
\text { group }\end{array}$ & $\begin{array}{l}\text { Group-I } \\
\text { Group-II }\end{array}$ & $\begin{array}{l}24(12.2) \\
8(13.6)\end{array}$ & $\begin{array}{l}172(87.8) \\
51(86.4)\end{array}$ & $\begin{array}{l}1.12(0.50,2.65) \\
1.00\end{array}$ & \\
\hline
\end{tabular}

$* *$ significant $(\mathrm{P}<0.01)$ in multivariate analysis

Note: Departments had been categorized based on the closeness to transfusion science as group-I comprises Health officer,

Anesthesia, Nurse, Midwifery and Medical Laboratory Sciences; Group-II comprises physiotherapy, Pharmacy, Optometry and EHOHS

\section{DISCUSSION}

Sustaining the necessary level of blood supply is the core concern of many organizations working on health care facility. For this reason, identifying the level of knowledge, attitude and practice is crucial. An attempt was made to assess the knowledge, attitude, practice regarding blood donation and to identify its associated factors among graduating undergraduate Health Sciences students at the College of Medicine and Health Sciences, University of Gondar.

This study showed that $123(48.2 \%)$ of the study participants were knowledgeable about blood donation. This is comparable with a study conducted in Central India (52.5\%) (16). However, the finding is lower than studies done in Nigeria $(85 \%)$ (20), Thailand (80\%) (18), South India (62\%) (17), Addis Ababa, Ethiopia (83.6\%) (21) and Adama, Ethiopia (79.4\%) (22). On the other hand, it is higher than the findings of studies done in Nepal (32.4\%) (12), South India (35.65\%) (15), Manipur (9\%) (23) and Kollam, Kerala (35\%) (24). The possible reason for the variation might be attributed to the differences in sociodemography and access to learning opportunities on the importance of blood donation.

This study also revealed that nearly $80 \%$ of the students had a positive attitude regarding blood donation. This finding is lower than study conducted in Pondicherry, India, in which $85 \%$ of the participants had positive attitude and were willing to donate blood voluntarily (19). Moreover, it is lower than study done in South Indian (25) in which $87.3 \%$ of the respondents showed favorable attitude about blood donation. However, this finding is higher than the study conducted on Addis Ababa University Health Science students, in which $68 \%$ of the respondents had a favorable attitude (21). This difference might occur due to socio-cultural differences and educational attributes between the respondents. This indicates the importance of incorporating blood donation in the Health Sciences Curricula.

This study showed that $12.5 \%$ of graduating undergraduate Health Science students had ever donated blood. This is comparable to studies done in Thailand (11\%) (18), Tamil's ado, South India, $(10.75 \%)(15)$, South India $(12.76 \%)$ (13) and Nigeria (15\%) (20). However, it is lower than studies conducted in Central India (47.5\%) (9), University of South India (38\%) (17), and Larissa, Greece (23.9\%) (26). These differences might be due promotional effect of the blood banks and other social institutions regarding the importance of blood donation.

In this study, nearly half of the study participants were knowledgeable, and nearly $80 \%$ 
of them had positive attitude regarding blood donation. However, a small proportion of them had ever donated blood before, $12.5 \%$. This is comparable with other study conducted in Thailand where $80 \%$ of the study participants had good knowledge, but only $11 \%$ of them had donated blood before (18). Therefore, the finding of this study would suggest that knowledge does not necessarily lead to actual blood donation practice because of the mythical beliefs and wrong perception still held by the community where participants lived with (20).

The main reasons that the study participants reported for not donating blood were fear of pain $(31.8 \%)$, lack of adequate information $(24.3 \%)$ and had not been asked yet to donate $(22.8 \%)$. The finding of this study was in agreement with previous studies $(12,13,18,20)$.

In this study, students aged $\geq 25$ years had statistically significant practice of blood donation. The possible reason might be the fact that, as individuals get mature, they feel more socially responsible. Besides, they do have more access to information, which may decrease the feeling of fear related to blood donation. Therefore, to increase the number of volunteer blood donors, undergraduate Health Science students should be constantly encouraged to donate blood through different blood campaigns.

Large proportion of graduating undergraduate Health Science students had a positive attitude regarding blood donation, but the practice of blood donation was low. The knowledge of students about blood donation is low compared to the expected knowledge since their profession is related to blood Transfusion Science and as they are graduating students. Age was significantly associated with practice of blood donation. Therefore, promotion and sanitization strategies to enhance knowledge, attitude and practice of students concerning blood donation is essential. Even though there are many higher educational institutions across the country, there is a shortage of potential blood donors to meet the safe blood requirements of the country. The role of healthcare educational institutions and that of their students in voluntary blood donation is crucial. They should play a leading role in donating blood and creating awareness among the entire students of the institution. Hence, targeted strategies such as seminars, trainings and workshops should be regularly conducted to increase awareness and encourage voluntary blood donation among student.

The major limitations of this study were related to the inherent nature of knowledge, attitude and practice studies: first, the responses of the students might be influenced by socially desirable traits, so that the attitude and practice of the students may not be exactly reflected; and second, there is a possibility of recall bias while responding to some of questions. The other limitation of this study is that the students were from only one Medical and Health Sciences College; hence the result is not generalizable to the students of all Medical and Health Science Colleges.

\section{ACKNOWLEDGEMENTS}

We would like to thank the College of Medicine and Health Sciences of the University of Gondar for its material support. Our special thanks and appreciation goes to the study participants who voluntarily participated in this study. We also thank School of Biomedical and Laboratory Sciences for the technical and material support provided during the study.

\section{REFERENCES}

1. Action Plan for blood safety. National AIDS Control Organization, Ministry of Health and Family Welfare, Government of India, New Delhi. 2007.

2. WHO. Towards $100 \%$ Voluntary Blood Donation: A Global Framework for Action. 2010. Available at:http://www.who.int/bloodsafety/publications/97 89241599696/en/. Accessed on February 10, 2017.

3. Karim AM, Admassu K, Schellenberg J, et al. Effect of Ethiopia's health extension program on maternal and newborn health care practices in 101 rural districts: a dose-response study. PloS one. 2013:8(6): e65160.

4. WHO. Safety WB, Availability-Fact sheet N. 279. 2014. Available at: http://www.searo.who.int/thailand/factsheets/fs002 9/en/. Accessed January 10, 2017. 
5. Abolfotouh A, Al-Assiri MH, Al-Omani $\mathrm{M}, \mathrm{Al}$ Johar A, Al Hakbani AS, Alaskar AS. Public awareness of blood donation in Central Saudi Arabia. Int J Gen Med. 2014; 7:401.

6. Ingale L, Gessesse S, Miruts G. Magnitude and factors associated with voluntary blood donation practice among adult Mekelle population, north Ethiopia: a community-based cross-sectional study. Research and Reviews. J Oncol Hematol. 2015; 4(3):5-13.

7. Melku M, Terefe B, Asrie F, et al. Knowledge, Attitude, and Practice of Adult Population towards Blood Donation in Gondar Town, Northwest Ethiopia: A Community Based Cross-Sectional Study. Journal of Blood Transfusion. 2016;2016:794986

8. Alfouzan N. Knowledge, attitudes, and motivations towards blood donation among King Abdulaziz Medical City population. Int J Family Med. 2014; $1: 8$.

9. Merav B, Lena G. Investigating the factors affecting blood donation among Israelis. Int Emerg Nurs. 2010; 19(1):37-43.

10. ENSA. About $40 \%$ of Ethiopian Mothers Die because of Lack of Enough Blood Donors. Cantabria, Spain: ENSA; 2014.

11. WHO. Ethiopia Commences World Blood Donor Day 2014 Celebrations. Geneva, Switzerland: WHO; 2014.

12. Mrigendral A. Study on Knowledge, Attitude and Practice of Blood Donation among Students of Different Colleges of Kathmandu, Nepal. International Journal of Pharmaceutical \& Biological Archives. 2013;;4(3):424-28.

13. Yerpude PN, Jordan KS. A cross-section study on KAP on blood donation Among medical students South India. Int $J$ Med health Sci. 2013;2(4):382-5.

14. Agrwwa A, TiwariA, Ahuja A. KAP of people toward voluntary blood donation in uttarakhand, Asian. J Transfus, Sci. 2013;7(1):59-62.

15. Manikandan.S, Srikumar R, Ruvanthika PN. A study on KAP on blood donation Among health profession student in Chennai, Tamil's ado, south India. International of scientific and research publication. 2013;3(3):2-4.

16. Geri PA, Pale DB. Knowledge and Attitude about Blood Donation Amongst Undergraduate Students of Gravari Institute of Medical Sciences Deemed
University of Central India. Ann Troop Med Public Health. 2012;5:569-73.

17. Saba KM, Remy A, Binue VS, Vive R. Knowledge, Attitude and Practice on Blood Donation among Health Science Students in a University campus, South India. Health Allied Sacs J. 2011;10(2):6.

18. Wiwanitkit V. Knowledge about blood donation among a sample of Thai university students. Vox Sang. 2002;83(3):97-9.

19. BhaRatWaj RS, Vijaya K, RajaRam P. A Descriptive Study of Knowledge, Attitude and Practice with regard to Voluntary Blood Donation among Medical Undergraduate Students in Pondicherry, India. Journal of Clinical and Diagnostic Research. 2012; 6(4): 602-604.

20. Salaudeen AG, Odeh E. Knowledge and behavior towards voluntary blood donation among students of tertiary institution in Nigeria. Nigerian Journal of Clinical practice. 2011;14(3):3.

21. Misganaw C, Tenkir M, Deresa A, Tesfaye M, Tessema TT, Taye H. The level and associated factors of knowledge, attitude and practice of blood donation among health science students of Addis Ababa University. IJMHSR. 2014;1(10):105-18.

22. Woldeab H, Olana R and Garoma S. Knowledge, attitude and practice of students towards blood donation in Arsi University and Adama Science and Technology University: a comparative cross sectional study. BMC Hematology. (2017) 17:20.

23. Syiemlieh AJ, Akoijam BS, Kumar S. Assessment of Knowledge, Perception \& Practice of Voluntary Blood Donation among Health Professional Students in RIMS, Imphal, Manipur. IOSR-JDMS. 2016; 15(10):26-30.

24. Ahmad NA, Abraham J, Sony S, et al. Assessment of knowledge, attitude and practice (KAP) of blood donation among MBBS students of a medical college in Kollam, Kerala. Jemds.2015;887.

25. Karakkamandapam S, Raghavan V, Sabu KM. Knowledge, attitude and practice on blood donation among health science students in a university campus, South India. OJHAS. 2011; 10(2):1-3.

26. Papagiannis D, Rachiotis G, Symvoulakis EK, et al. Blood donation knowledge and attitudes among undergraduate health science students: A crosssectional study. Transfus Apher Sci. 2016; 54(2):303-8.

DOI: http://dx.doi.org/10.4314/ejhs.v28i5.8 